\title{
Arthroscopic Soft Tissue Releases of the Knee
}

\author{
Michael R. Chen and Jason L. Dragoo \\ Stanford University Department of Orthopaedic Surgery,
}

USA

\section{Introduction}

Intra-articular fibrosis, which includes a family of disorders such as anterior interval scarring, posterior capsular contracture, a tight lateral retinaculum, as well as arthrofibrosis, may lead to alterations in joint biomechanics and can result in pain. 1, 2, 3 Specific releases for each region of fibrosis, along with characteristic physical examination findings, have been described. Familiarity with the diagnosis and arthroscopic treatment of these disorders may lead to improved treatment outcomes in this patient population.

\section{Anterior interval release}

\subsection{History and physical examination}

The anterior interval has been defined as the space between the infrapatellar fat pad and patellar tendon anteriorly, and the anterior border of the tibia and transverse meniscal ligament posteriorly.1 Trauma or previous surgery may cause hemorrhage or inflammation of the fat pad (Hoffa's Syndrome), which may be followed by fibrosis. If fibrosis occurs between the fat pad and transverse ligament or anterior tibia, it will lead to dysfunction of the anterior knee structures, such as decreased excursion of the patellar tendon and result in stretching of the surrounding synovial tissue, which may lead to pain or even loss of knee extension. 1 Fibrosis within the anterior interval can exist on a spectrum of severity. Paulos et al. described infrapatellar contracture syndrome, a severe form of anterior interval scarring, with fibrosis of the fat pad and severe limitations in range of motion. 5

Patients with anterior interval scarring complain of anterior knee pain, and frequently describe a sense of fullness within the knee, especially with extension. Physical examination may demonstrate a small flexion contracture, decreased proximal excursion of the patella, as well as a positive Hoffa's test 1 . Hoffa's test is performed by placing the thumb at the margin of the infrapatellar fat pad and patellar tendon medially and laterally with the knee flexed at $30^{\circ}$ (Figure 1). Pressure is applied with the thumb, and the knee is fully extended. Increased pain in the fat pad with knee extension indicates a positive result. Patients may also have pain in the fat pad with forceful hyperextension of the knee. The patellar tendon and patella should also be carefully examined to ensure they are not also causes of the anterior knee pain.

Scarring in the fat pad can be visualized on standard T1- and T2-weighted magnetic resonance imaging (MRI). Low T1- and T2- signal identified on sagittal images coursing from the posterior portion of the fat pad to the anterior surface of the tibia and/or transverse meniscal ligament indicates anterior interval scarring. 


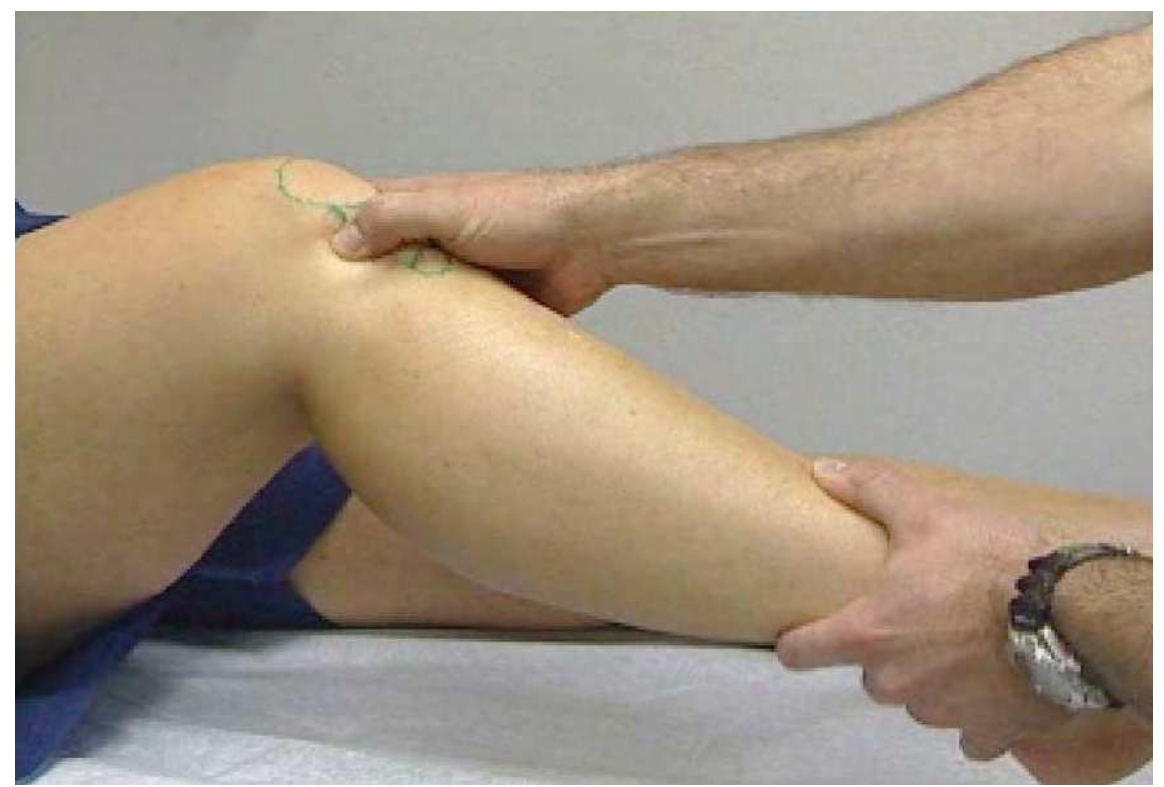

Figure 1A

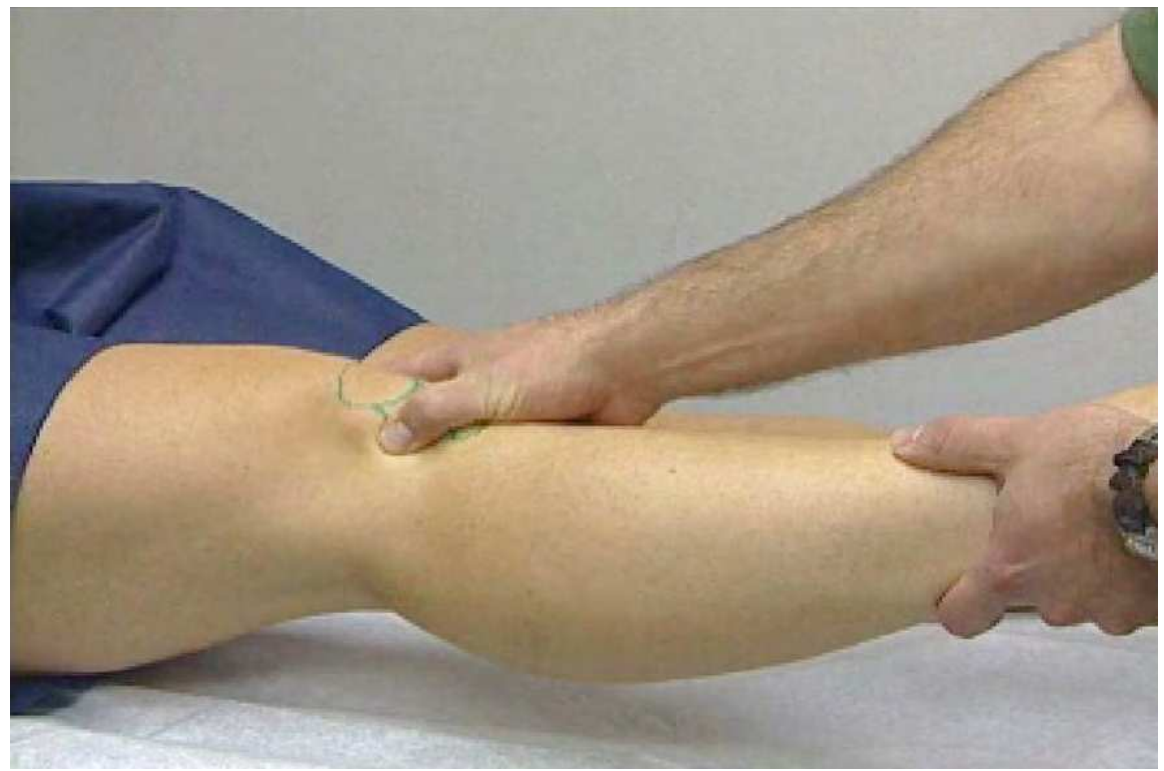

Figure 1B

Fig. 1. Demonstration of Hoffa's test. A, The thumb is placed at the margin of the infrapatellar fat pad and the patellar tendon with the knee in $30^{\circ}$ of flexion. B, Pressure is applied with the thumb and the knee and then brought into full extension. Increased pain in the fat pad indicates a positive result. 


\subsection{Anterior interval release}

Arthroscopic treatment of anterior interval scarring begins with establishment of a modified anterolateral viewing portal. The portal is established slightly more lateral and proximal to the standard placement to allow easier visualization of the anterior interval structures (Figure 2). Viewing of anterior interval scarring with the $30^{\circ}$ arthroscope will demonstrate decreased opening of the interval with extension as well as fibrosis of the infrapatellar fat pad.

Fibrosis can be released with use of a $70^{\circ}$ electrothermal probe via a modified anteromedial portal (Figure 2). Systematic release begins anterior to the transverse ligament, starting just anterior to the anterior horn of the medial meniscus, and proceeding laterally and anterior to the anterior horn of the lateral meniscus (Figure 3). The release is continued until the anterior tibial cortex in encountered or until normal fat pad tissue is seen. Adequate release is confirmed by visualization of interval widening with knee extension and closing with flexion. Care is taken to avoid disrupting the anterior meniscal attachments or the transverse meniscal ligament. Meticulous hemostasis is obtained to prevent postoperative bleeding and recurrent scarring 1 .

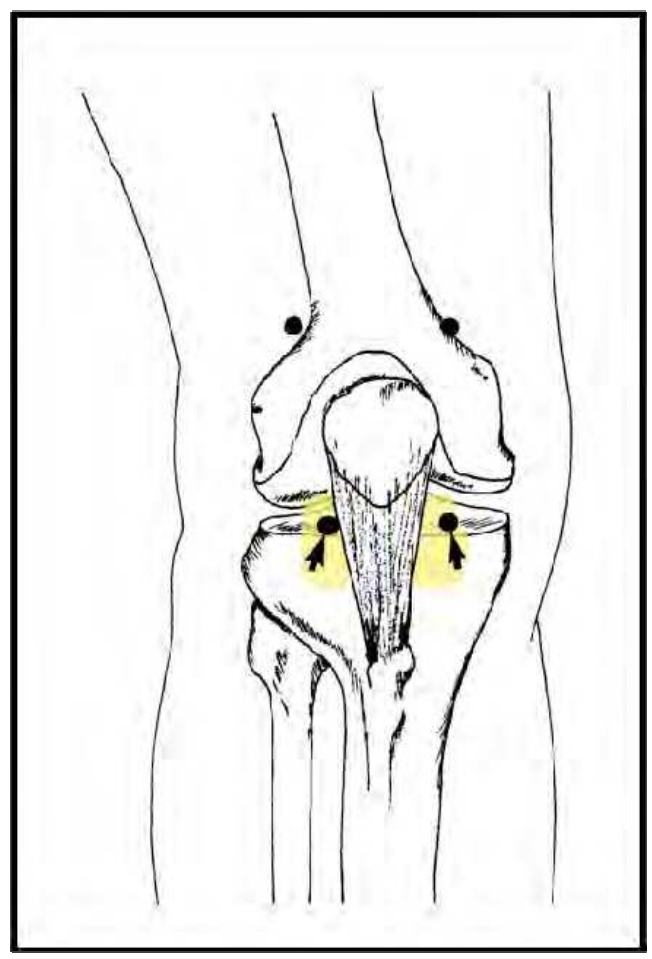

A

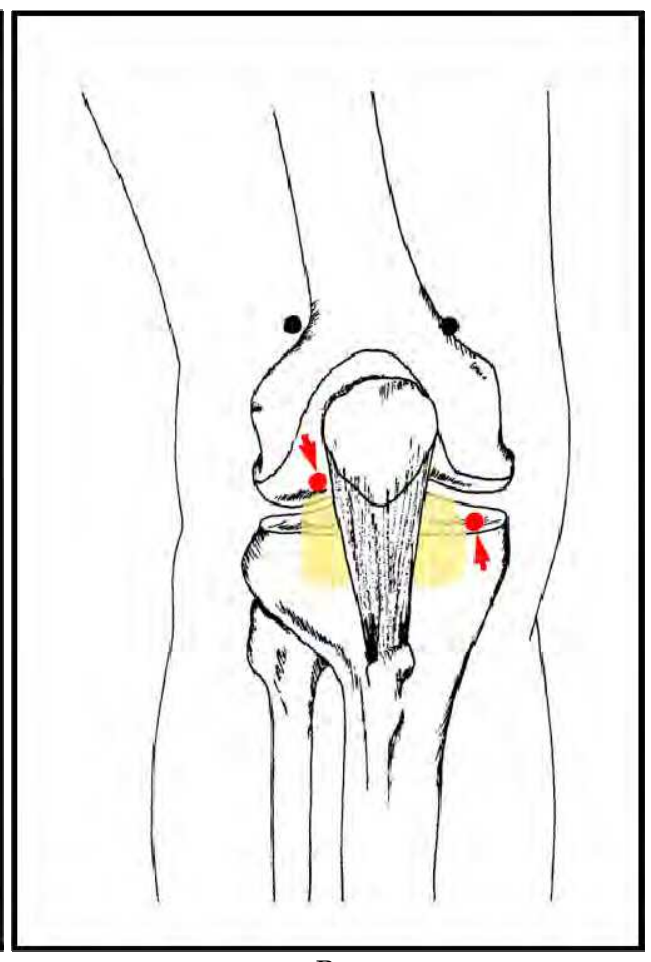

B

Fig. 2. Modified arthroscopic portals for anterior interval release. A, Standard arthroscopic portals. B, The modified anterolateral portal established slightly more lateral and proximal to the standard placement to allow easier visualization of the anterior interval and the modified anteromedial portal established more medial to allow instrumentation of the anterior interval. 


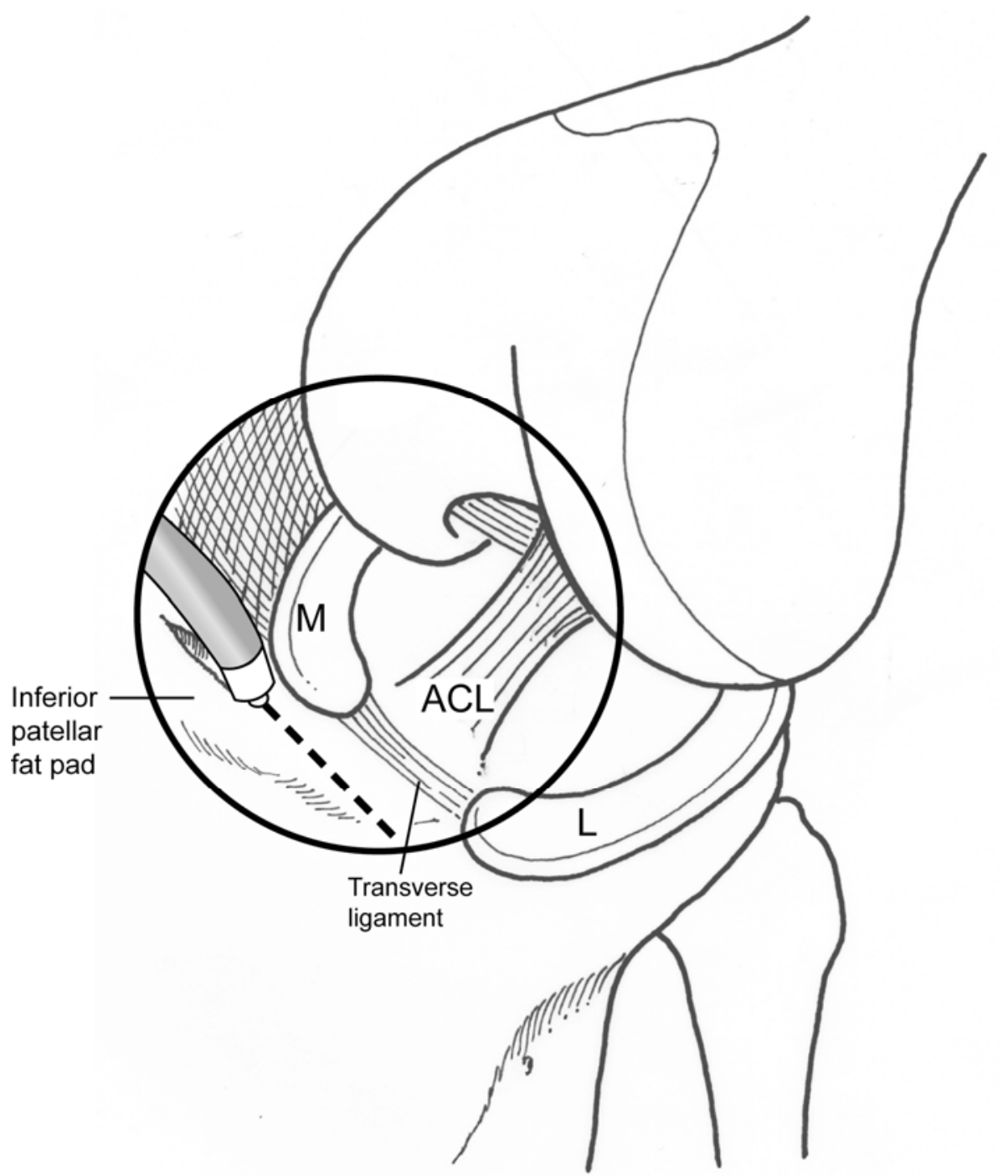

Fig. 3. Location of anterior interval release. The release is made anterior to the transverse meniscal ligament and proceeds anterior to the anterior horn of the medial and lateral meniscus.

Postoperatively, the goal is to prevent scar reformation, while preserving joint mobility. Initially, rehabilitation is focused on establishing full range of motion and patellar and patellar tendon mobility. Patients are limited to touch-down weight bearing for 2 weeks to limit inflammation and to maximize interval excursion. 4 Patients are also usually prescribed anti-inflammatory medications for 4 to 6 weeks following surgery. After 6 weeks, the goal is the return functional strength followed by sports specific exercise and gradual return to sport. 


\subsection{Results}

Steadman et al 1 described the results of isolated anterior interval release in twenty-five consecutive patients. Patients failed a minimum of six months of physical therapy and nonsteroidal anti-inflammatory medications. Following arthroscopic release, the average Lysholm score significantly improved from 59 preoperatively to 81 postoperatively, while the average International Knee Documentation Committee (IKDC) score improved from 49 to 70. Four patients had failed results, requiring a second surgical release. Ogilvie-Harris and Giddens described arthroscopic resection of the infrapatellar fat pad in patients with Hoffa's syndrome. 6 Patients had significant improvement in symptoms and function after surgery at an average follow-up of 76 months.

Conversely, patients with severe scarring, or infrapatellar contracture syndrome, had symptoms of patellofemoral arthritis and tibiofemoral arthritis and could not return to preinjury sport or work despite improvements in range of motion after arthroscopic and open treatment. 5

\section{Posterior capsular release}

\subsection{History and physical examination}

Flexion contractures are one of the main factors that adversely affect patient outcome and knee function following surgery. 5, 7, 8 A 5 o loss of extension can cause a noticeable limp during ambulation and patellofemoral irritability, and a loss of $10^{\circ}$ is poorly tolerated. 3 Deficits greater than $20^{\circ}$ result in a significant leg-length discrepancy. 8 Contracture of the posterior capsule is the primary cause of extension loss, however anterior interval scarring, patellar entrapment, anterior cruciate ligament (ACL) graft malposition and hamstring tightness can also contribute. 5, 7, 8, 9

Patients with an extension deficit should be systematically evaluated for the multiple potential causes of a flexion contracture. A thorough history of any trauma or surgical procedures should be obtained. Knee range of motion should be carefully measured and the quality of the endpoint should be recorded. Flexion contractures exhibiting a firm endpoint tend to involve the posterior capsule, while a spongier endpoint typically indicates involvement of the patellofemoral mechanism or anterior interval.

MRI is the imaging modality of choice. Thickening or scarring of the posterior capsule, indicated by low T1 and T2 signal, may sometimes be observed.

\subsection{Posterior capsular release}

Surgical intervention is indicated in a patient with a 10-15 degree flexion contracture with an unyielding endpoint that has failed non-operative management, 10 however even smaller degrees of contracture may not be tolerated in the elite athletic population. Both open 9,10,11 and arthroscopic posterior capsular releases 12,13 have been described, however arthroscopic releases are more technically demanding.

The patient is positioned for standard arthroscopy, however the contralateral leg is placed in a well-leg holder and the foot of the bed is dropped. Care should be taken to place the tourniquet high on the thigh to allow adequate draping for the creation of a posteromedial and/or posterolateral portal (Figure 4). An arthroscopic pump is generally recommended to maintain a constant intra-articular pressure and ensure distension of the posterior capsule. Anterolateral and anteromedial arthroscopic portals are created near the edge of the patellar tendon to allow instrumentation to be used in the posterior compartment. 
The posteromedial compartment is visualized with a $30^{\circ}$ arthroscope via the anterolateral portal by placing it between the medial femoral condyle and posterior cruciate ligament (PCL). 12 Often, a blunt arthroscopic obturator must first be used to break through scar tissue or adhesions, prior to inserting the arthroscope. $12 \mathrm{~A}$ spinal needle is placed under direct visualization from the posteromedial skin through the posteromedial capsule to localize the posteromedial arthroscopic portal (Figure 4). The portal is then created by making a small incision and advancing an arthroscopic cannula through the incision into the posteromedial compartment under direct visualization. Similarly, the arthroscope may be placed between the ACL and lateral femoral condyle to visualize the posterolateral compartment. A posterolateral portal can be created in a similar manner; however care must be taken to avoid the peroneal nerve, which lies on the posteromedial border of the biceps tendon. Incision must be anterior to the biceps and blunt dissection must be performed during portal placement.

Two methods of arthroscopic capsular release have been described. Laprade et al. 12 described an isolated posteromedial release for flexion contractures. Separation of the posteromedial capsule from the posteromedial structures, including the medial gastrocnemius tendon and muscle, using a blunt arthroscopic obturator or a small periosteal elevator was recommended. An arthroscopic basket punch and shaver, with the shaver blade facing anteriorly, are then used to release the posteromedial capsule. The procedure

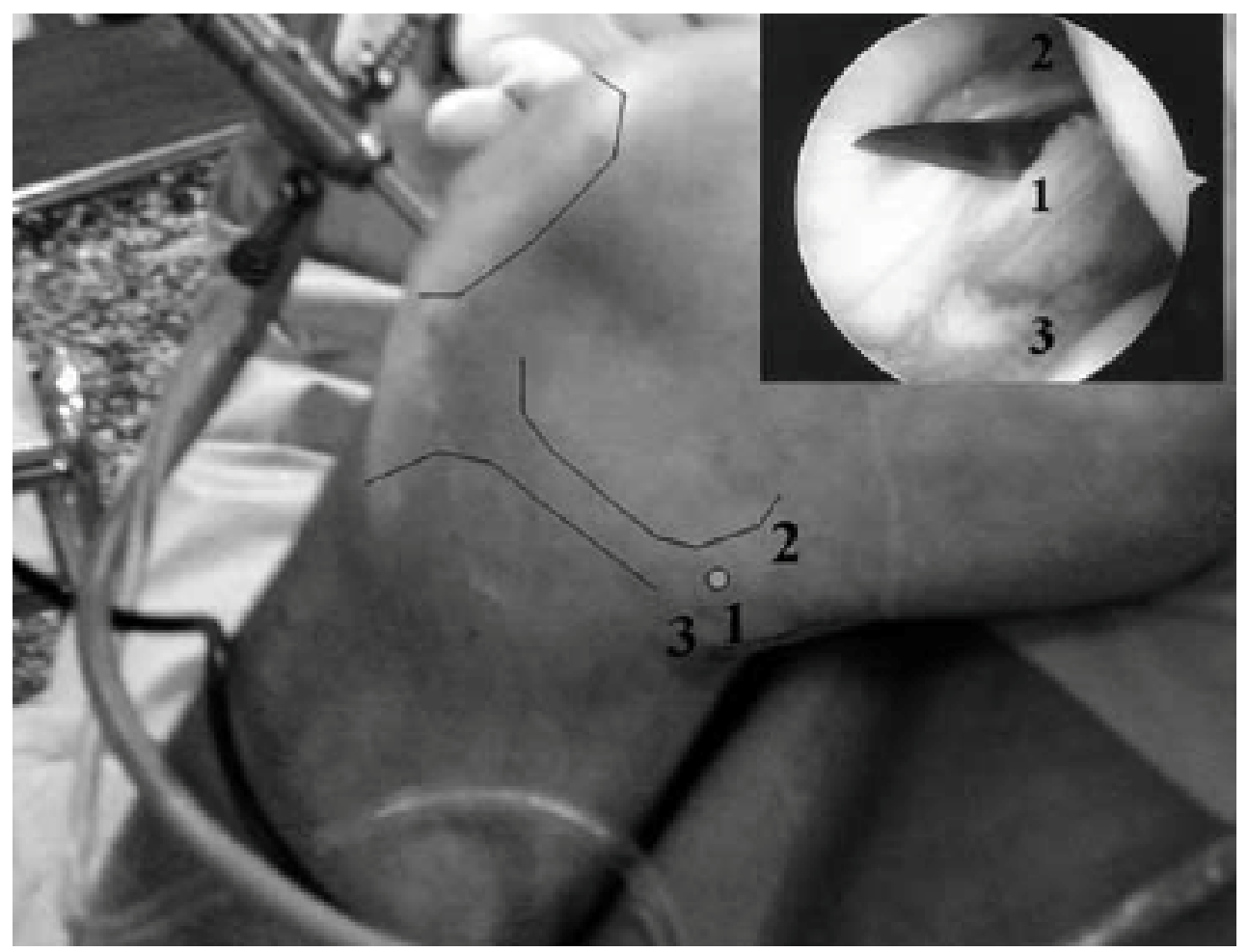

Figure 4A 


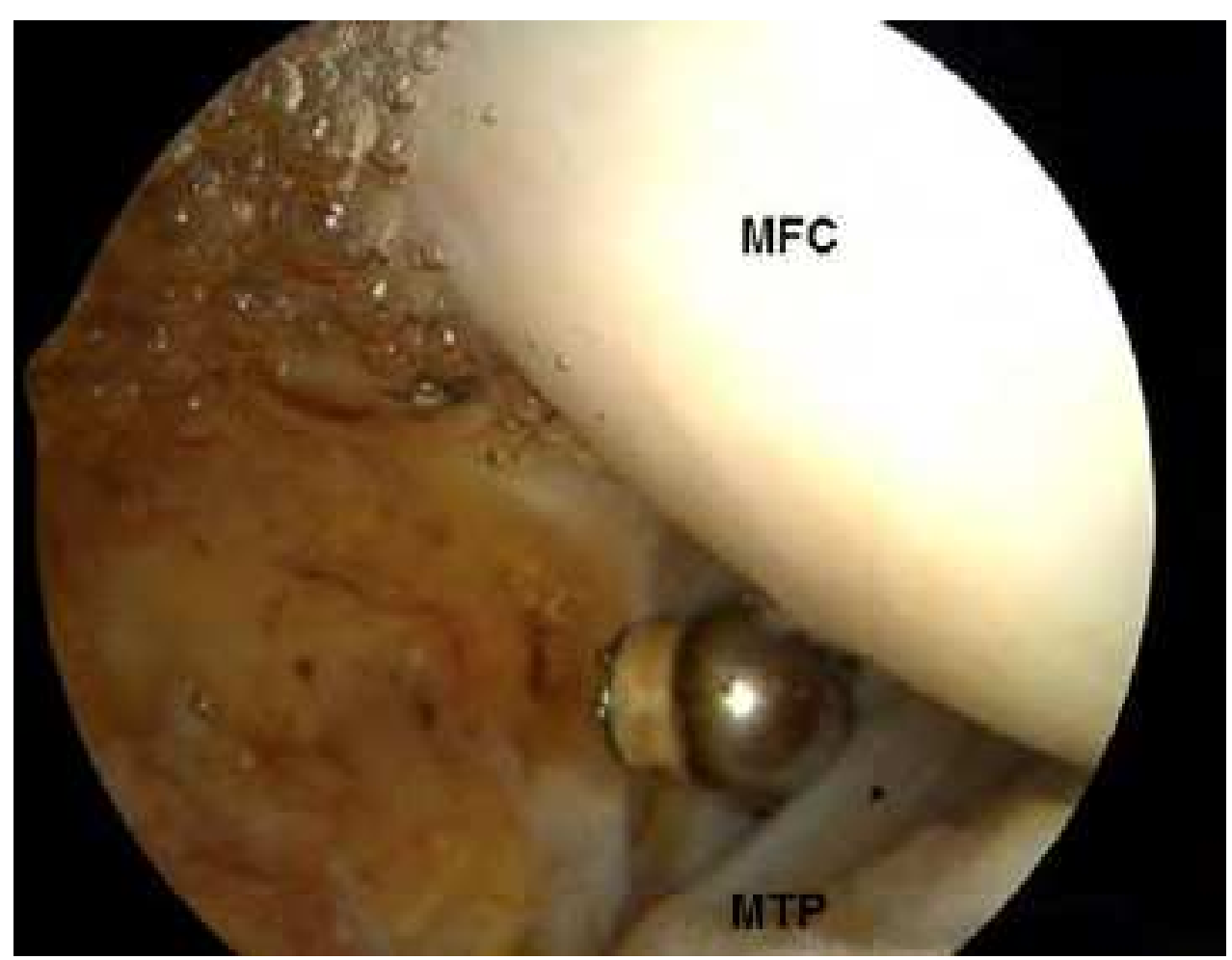

Figure 4B

Fig. 4. Posteromedial arthroscopic portal. A, The entry site (1) is between the posterior medial femoral condyle (2) and posterior medial tibial plateau (3) and created under direct visualization. B, View from the posterolateral portal toward the posteromedial aspect of the knee during capsular release. MFC: medial femoral condyle. MTP: medial tibial plateau.

starts medially and proceeds laterally to the midline, in line with the PCL at the midcapsular level. The gastrocnemius muscle and tendon become visible as the capsule is released.

Mariani 13 described posteromedial and posterolateral arthroscopic capsular releases. The arthroscope may be placed in either posteromedial or posterolateral portals to visualize the contralateral compartment. Often there is a posterior septum that must first be released. A blunt trocar may be used to perforate the septum and then expanded with a shaver or radiofrequency probe. Posterior adhesions are removed until the femoral condyles become visible. A punch or radiofrequency probe is then directed toward the superior capsular attachments at the condyle. The capsule is then progressively released. The tendon of the gastrocnemius is then recognized and can also be released to allow for a greater posterior release.

Postoperatively, patients may be admitted to the hospital for pain management and initial physical therapy. An indwelling epidural catheter is often beneficial, in addition to oral and 
intravenous analgesics. Early, aggressive physical therapy is begun on the first postoperative day. A continuous passive motion machine is used and is alternated in cycles with extension splinting. Patients are advanced to weight bearing as tolerated and weaned from crutches when they are able to ambulate pain free. Patients are also placed on deep venous thrombosis prophylaxis for two weeks. At discharge, patients continue with outpatient physical therapy and nighttime extension splinting for at least 6 weeks.

\subsection{Results}

Laprade et al 12 reported the results of isolated arthroscopic posteromedial release in 15 patients with an average follow-up of 24 months. Preoperative knee extension averaged $15^{\circ}$ and significantly improved to $0.7^{\circ}$ at the final follow-up.

Mariani 13 reported the results of arthroscopic posterior capsular release in 18 patients. Extension deficits averaged $34^{\circ}$ preoperatively and improved to $3^{\circ}$ at final one year followup. Patients with more severe pre-operative flexion contractures need more aggressive releases of both the posteromedial and posterolateral capsule and possibly partial release of the gastrocnemius tendon if the results are not adequate.

\section{Lateral retinacular release}

\subsection{History and physical examination}

The patellofemoral joint is an intrinsically unstable articulation, stabilized dynamically by the vastus medialis obliqus (VMO) and statically by the medial patellofemoral ligament and lateral retinaculum. 14, 15, 16 Imbalance may arise because of weakness in the VMO or tightness from the lateral retinaculum. Axial alignment and bony morphology of the patellofemoral joint may also contribute to this imbalance.

Evaluation of anterior knee pain begins with a history of aggravating factors such as inclined ambulation, squatting, prolonged sitting, or going up and down stairs. If present, patellar instability needs to be identified, as this can affect surgical decision making.

Physical examination begins with inspection of patellar glide, or excursion, which should be examined in all directions. 17 The VMO can be inspected for atrophy or weakness, and the medial and lateral patellar facets should be palpated for tenderness. Patellar tilt should be carefully assessed. 17 The inability to elevate the lateral aspect of the patella to neutral with the knee in full extension and the patella centered in the trochlea indicates a tight lateral retinaculum and possible over-constraint of the patellar mechanism (Figure 5). A patellar apprehension test should also be performed to evaluate for instability at $30^{\circ}$ of flexion.

Radiographic evaluation consists of standard weight bearing AP and lateral radiographs of the knee, as well as an axial view of the patellofemoral joint in $30^{\circ}$ or $45^{\circ}$ of flexion. Patellar tilt and subluxation, as well as trochlear and patellar bony morphology can be evaluated on the axial view. MRIs are not routinely indicated for isolated patellofemoral pathology unless the articular surface warrants evaluation or there is history of patellar instability.

Initial, non-operative treatment generally begins with physical therapy. Specific therapy is aimed at strengthening of the hip external rotators and abductors, VMO and core musculature, as well as patellar mobilization. Patellar taping using the McConnell method may also be beneficial. 18 Surgical intervention is only indicated after failure of nonoperative management. 


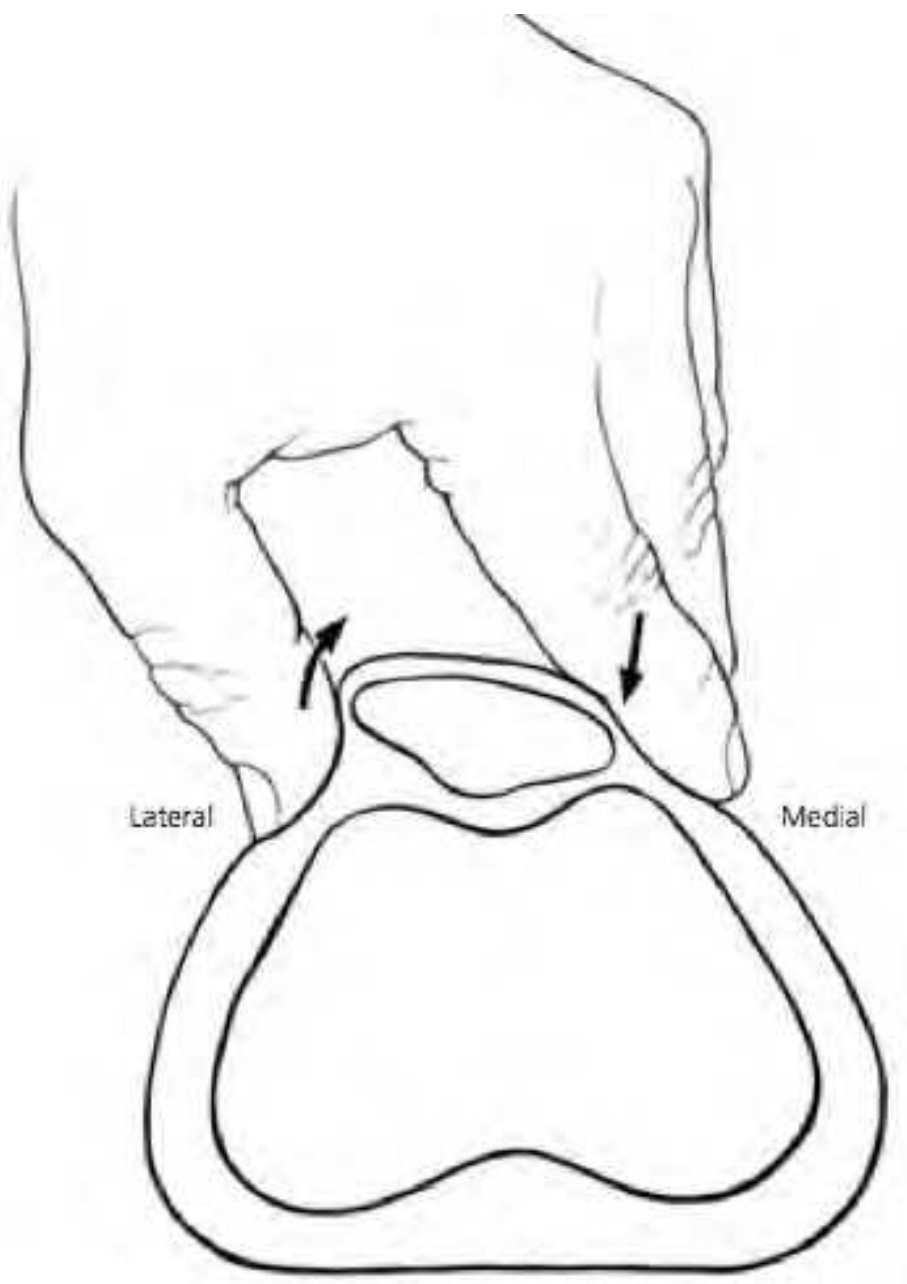

Fig. 5. Patellar tilt test. The patella is centralized in the femoral trochlea with the knee in full extension. The medial and lateral aspect of the patella is stabilized and the lateral aspect is elevated. Inability to elevate the lateral aspect of the patellar to neutral indicates a tight lateral retinaculum.

\subsection{Lateral release}

Lateral release is indicated in patients who have failed physical therapy and have increased lateral facet pressure demonstrated either radiographically or clinically with a tight lateral retinaculum on tilt testing. 2,18,19 Isolated lateral release is contraindicated for patellar instability. 19,20,21

A tight lateral retinaculum is confirmed on patellar tilt testing once the patient is under anesthesia. 20 Patellar tilt and areas of chondromalacia are also visually assessed and documented arthroscopically. 
Modern lateral releases are generally performed using an arthroscopic electrothermal probe to aid in hemostasis, since hemarthrosis is the most common complication of this procedure. 23 The anterolateral portal is often used as the working portal and the anteromedial portal as the viewing portal. Placement of the electrothermal probe through a superior portal is possible, but usually unnecessary. The lateral release is performed approximately $1 \mathrm{~cm}$ posterior to the lateral border of the patella to avoid devascularization. A complete release begins at the level of the proximal pole of the patella and is continued distally to the level of the distal pole. Modified releases begin distal to the vastus lateralis insertion on the patella and are continued distally only far enough to achieve a neutral tilt test. The release is performed in layers to prevent over-release.

Complications of lateral releases are common, especially with excessive release, which continues beyond the fat and muscle layers or disrupts the vastus lateralis tendon insertion. Over-release can result in wound complications or medial patellar instability. Hemarthrosis is the most common complication; therefore meticulous hemostasis is required due to the proximity of the geniculate arteries. 23

Weight-bearing is generally limited for several days to decrease the incidence of hemorrhage and inflammation. Bracing after lateral release is not routinely used. Patellar mobilization is begun immediately after surgery in physical therapy, followed by quadriceps strengthening.

\subsection{Results}

Several studies have demonstrated $60-90 \%$ satisfactory results with arthroscopic lateral release for patellofemoral pain with maltracking and without instability. 22,23,24,25 Increasing amounts of chondromalacia and instability were associated with less favorable results in these studies. When arthroscopic lateral release has been performed for indications other than lateral patellar compression, the results have been poor. $19,20,21,22,25,27,28$

\section{Lysis of adhesions}

\subsection{History and physical examination}

Arthrofibrosis is the development of intra-articular scarring and adhesions due to trauma, previous surgery, prolonged immobilization and infection. In general, arthrofibrosis of the anterior structures of the knee cause of loss of flexion, while scarring of the posterior structures can cause loss of extension. Scarring and adhesions lead to loss of capsular compliance and pain. 29

Physical examination of the patella typically exhibits decreased excursion in all directions. Tenderness in the region of the supra-patellar pouch and/or infrapatellar fat pad is common, along with loss of range of motion.

If arthrofibrosis is recognized early, non-operative measures such as physical therapy modalities, range of motion exercises and anti-inflammatory medications may successfully improve motion. Manipulation under anesthesia has been a commonly performed procedure, however it is falling out of favor due to complications such hemarthrosis, which can predispose to further scar tissue formation, distal femur fractures and patellar tendon rupture. Arthroscopic lysis of adhesions avoids these complications and allows for controlled, focused treatment. 


\subsection{Arthroscopic lysis of adhesions}

A systematic evaluation as described by Kim, et al. allows for assessment and treatment of intra-articular sources of motion loss. 30 Capsular distension before arthroscopy is useful, as it re-establishes effective joint space, allows easier and safer insertion of instruments, enhances visualization, and may disrupt intra-articular adhesions. 31 Injection of sterile saline should be performed slowly to allow for capsular stretching and to avoid rupture of the capsule, preventing extravasation of fluid during arthroscopy. 29,31

Intra-articular volume capacity can be assessed by injecting the knee with 60cc's of sterile saline. 29,31 After injection, the 18 gauge needle is disconnected from the syringe. If the saline drips out of the needle, the capsule is under little tension and the intra-articular volume is considered normal (Figure 6B). If, however, the saline is expressed from the joint in a stream (Figure 6A), the capsule is under significant pressure indicating insufficient volume. The knee should then be evaluated for stuctures known to reduce interarticular volume. 29
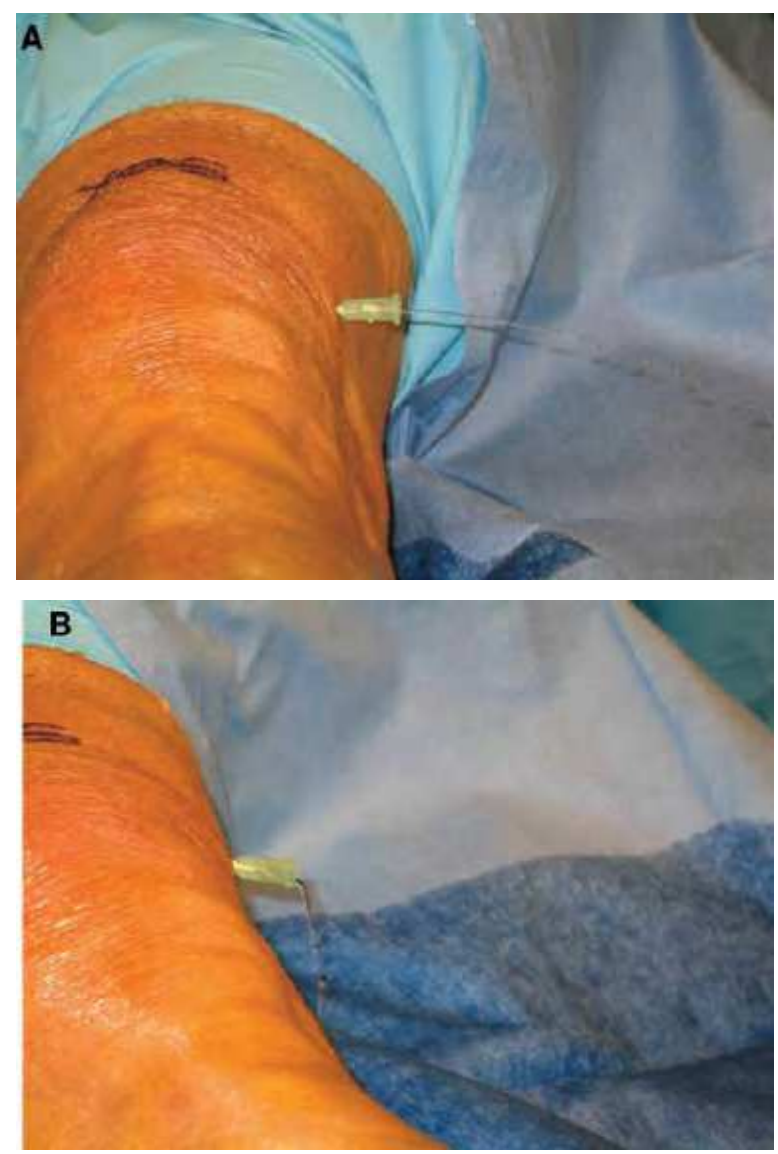

Fig. 6. Assessment of intra-articular volume. The preoperative knee is injected with $60 \mathrm{~mL}$ of sterile saline. A, Rapid outflow suggests insufficient intra-articular volume. B, Slow egress (drip) indicates normal volume. 
Using an electrothermal probe, adhesions are lysed and scarring is released to re-establish the suprapatellar pouch. Adhesions between the capsule and the femoral condyles are often observed and require release. The anterior interval is then re-established as necessary. 1 The medial and lateral patellar retinaculum are partially released if they are scarred, which improves patellofemoral mobility and capsular compliance. The intercondylar notch is then assessed for cyclops lesions.

After completing all anterior releases, the knee is then taken through a range of motion. If a persistent extension deficit remains, then the posterior compartment is assessed and released as previously described.

Postoperatively, an indwelling epidural catheter can help provide adequate pain management, which allows for immediate intensive physical therapy. Patients are placed in a continuous passive motion (CPM) machine immediately, and patellar mobilization and range of motion exercises are emphasized.

\subsection{Results}

Numerous authors have reported significant improvements of range of motion after lysis of adhesions from $35^{\circ}$ to $68^{\circ}$. $32,33,34,35$ The most common adverse outcome of this procedure is the inability to restore complete range of motion. 32,33,35 Several authors have also noted marked post-operative tenderness in the region of the infrapatellar fat pad, which resolved with non-operative measures. 32,33 Hematoma is rare with modern techniques using radiofrequency ablation and meticulous hemostasis during the procedure.

\section{Plica excision}

\subsection{History and physical examination}

Plicae are remnants of synovial membranes, which divide the embryologic knee into compartments. Plicae are traditionally described based on their anatomic location as suprapatellar, infrapatellar, and medial patellar or medial shelf. Lateral plicae have also been described, but are uncommon.

The suprapatellar plica is seen as a complete or partial synovial membrane that lies proximal to the proximal pole of the patella in the suprapatellar pouch. Arthroscopic studies have described an incidence of some form of suprapatellar plica as high as $87 \%$. 36 The infrapatellar plica originates in the intercondylar notch and inserts into the synovium around the infrapatellar fat pad. Posteriorly, it may be separate from the ACL or attached to it. It is commonly seen during arthroscopy, with an incidence up to $86 \%$. 36 The medial patellar plica originates on the medial wall of the knee, passes obliquely and inferiorly, sometimes crossing the suprapatellar plica, and inserts into the synovium surrounding the infrapatellar fat pad. The true incidence of the medial plica is unknown.

The presence of a plicae does not necessarily indicate a pathologic condition. However, plicae may become symptomatic if thickened, hypertrophic, inflamed and/or fibrotic. 37 The medial plica is most commonly pathologic, resulting in snapping or abrasion against the femoral condyle.

Pathologic plicae are notoriously difficult to diagnose because of their relative rarity, and shared symptoms with other more common knee pathology. There is often a history of trauma or repetitive stress, which may convert a non-pathologic plica into a symptomatic one. Patients mostly complain of pain in the location of the plica, which is usually exacerbated by activity, specifically kneeling or crouching. The incidence of swelling, 
clicking, or catching varies widely. Provocative meniscal tests and test for patellofemoral pathology are often positive, which further complicates the diagnosis. A thickened medial plica is occasionally palpable medially, just proximal to the joint line, which may be tender and felt to catch with flexion and extension. MRI can often demonstrate the presence of a plica, but not whether it is pathologic.

\subsection{Plica excision}

A standard diagnostic arthroscopy is always performed to ensure that other more common intra-articular pathologies are not present as the source of pain. Normal plicae appear soft and may be almost translucent at its edge and can be moved freely. In contrast, pathologic plicae often appear thickened and hypertrophic, while having the feel of a tight bowstring. Underlying chondral degeneration of the medial femoral condyle is often present with medial plica impingement.

When the diagnosis of a symptomatic plica is made, it should be resected along its entire length using an electrothermal probe. Care is taken to ensure that only the plica is resected, while protecting the surrounding structures.

The normal suprapatellar pouch extends to approximately $3-4 \mathrm{~cm}$ proximal of the proximal pole of the patella. With the arthroscope in the suprapatellar pouch, failure to visualize the quadriceps tendon suggests the presence of a complete suprapatellar plica dividing the suprapatellar pouch. The presence of an incomplete or complete suprapatellar plica can result in a decrease of knee volume, and pain. 29 Thus, suprapatellar plicae can be resected in patients with parapatellar pain with an electrothermal probe.

\subsection{Results}

The majority of results of arthroscopic plica excision have described medial plica excision and mostly been limited to small retrospective reviews. Kent et al. summarized the results of arthroscopic treatment of medial plica in studies published since 1980. 38 In all the studies reviewed, patients had $66 \%$ to $98 \%$ good to excellent outcomes with plica excision. Weckstrom et al. also recently described retrospective results of military recruits with arthroscopic medial plica resection at median 6-year follow-up. 39 Functional results as determined by the Kujala and Lysholm knee scores were good to excellent in $68 \%$ of patients.

Few reports exist of pathologic infrapatellar or suprapatellar plica. Demirag et al. 40 and Boyd et al., 41 in small series described $85 \%$ to $91 \%$ good or excellent results with arthroscopic resection of symptomatic infrapatellar plica, while Bae et al. 42 reported $90 \%$ good or excellent results with excision of a complete, symptomatic suprapatellar plica.

\section{Summary}

A myriad of inta-articular soft tissue disorders can cause significant morbidity within the knee. Arthroscopic techniques provide minimally-invasive efficacious alternatives to open treatment. Anterior interval release is a simple procedure for treating anterior interval scarring, a fibrotic condition still commonly unrecognized as a cause of anterior knee discomfort. Posterior capsular release, although technically demanding, is effective for treating flexion contractures secondary to scarring and contracture of the posterior capsule. Isolated lateral release provides satisfactory results for patellofemoral pain with maltracking without instability. Arthroscopic lysis of adhesions allows for controlled, focused treatment 
of all intra-articular causes of motion loss, with decreased risk of complications. Pathologic synovial plicae are an uncommon source of knee pain, but can be easily resected if symptomatic.

\section{References}

[1] Steadman JR, Dragoo JL, Hines SL, Briggs KK. Arthroscopic release for symptomatic scarring of the anterior interval of the knee. Am J Sports Med 2008 Sep;36(9): 17631769.

[2] Clifton, R, Ng CY, Nutton RW. What is the role of lateral retinacular release? J Bone Joint Surg Br 2010 Jan; 92(1):1-6.

[3] Sachs RA, Daniel DM, Stone ML, Garfein RF. Patellofemoral problems after anterior cruciate ligament reconstruction. Am J Sports Med 1989; 17(6):760-765

[4] Dragoo JL, Phillips C, Schmidt JD, Scanlan SF, Blazek K, Steadman JR, Williams A. Mechanics of the anterior interval of the knee using open dynamic MRI. Clin Biomech 2010 Jun; 25(5):433-437.

[5] Paulos L, Rosenberg T, Drawbert J, Manning J, Abbott P. Infrapatellar contracture syndrome: An unrecognized cause of knee stiffness with patella entrapment and patella infera. Am J Sports Med 1987 July-Aug;15(4): 331-341.

[6] Ogilvie-Harris DJ, Giddens J. Hoffa's disease: Arthroscopic resection of the infrapatellar fat pad. Arthroscopy 1994 April;10(2): 184-7.

[7] Saito T, Takeuchi R, Yamamoto K, Yoshida T, Koshino T. Unicompartmental arthroplasty for osteoarthritis of the knee: remaining postoperative flexion contracture affecting overall results. J Arthroplasty 2003 Aug;18(5): 612-618.

[8] Cosgarea AJ, DeHaven KE, Lovelock JE. The surgical treatment of arthrofibrosis of the knee. Am J Sports Med 1994 Mar; 22(2): 184-191.

[9] Lobenhoffer H, Bosch U, Gerich T. Role of posterior capsulotomy for the treatment of extension deficits of the knee. Knee Surg Sports Traumatol Arthrosc 1996;4(4) 237-241.

[10] Noyes FR, Barber-Westin SD in Noyes FR, Barber-Westin SD (eds.): Noyes' Knee Disorders: Surgery, Rehabilitation, Clinical Outcomes. Saunders, 2009, pp10531095.

[11] Millett P, Williams R, Wickiewicz T. Open debridement and soft tissue release as a salvage procedure for the severely arthrofibrotic knee. Am J Sports Medi 1999 SeptOct;27(5) 552-561.

[12] Laprade RF, Pedtke AC, Roethle ST. Arthroscopic posteromedial capsular release for knee flexion contractures. Knee Surg Sports Traumatol Arthrosc 2008 May;16(5) 469475 .

[13] Mariani PP. Arthroscopic release of the posterior compartments in the treatment of extension deficit of knee. Knee Surg Sports Traumatol Arthrosc 2010 Jun;18(6) 736-741.

[14] Conlan T, Garth WP Jr, Lemons JE. Evaluation of the medial soft-tissue restraints of the extensor mechanism of the knee. J Bone Joint Surg Am 1993; 75-A:682-693.

[15] Fulkerson JP, Gossling HR. Anatomy of the knee joint lateral retinaculum. Clin Orthop 1980;153:183-188.

[16] Merican AM, Amis AA. Anatomy of the lateral retinaculum of the knee. J Bone Joint Surg Br 2008; 90-B: 527-534.

[17] Post WR. Clinical evaluation of patients with patellofemoral disorders. Arthroscopy 1999 Nov;15(8): 841-851. 
[18] Fulkerson JP. Diagnosis and treatment of patients with patellofemoral pain. Am J Sports Med 2002; 30(30): 447-456.

[19] Panni AS, Tartarone M, Patricola A, Paxton EW, Fithian DC. Long-term results of lateral retinacular release. Arthroscopy 2005;21:526-531.

[20] Colvin AC, West RV. Patellar Instability. J Bone Joint Surg Am 2008;90:2751-2762.

[21] Lattermann C, Toth J, Bach BR Jr. The role of lateral retinacular release in the treatment of patellar instability. Sports Med Arthrosc 2007;15:57-60.

[22] Grana WA, Hinkley B, Hollingsworth S. Arthroscopic evaluation and treatment of patellar malalignment. Clin Orthop Relat Res 1984 Jun; 186: 122-128.

[23] Ogilvie-Harris DJ, Jackson RW. The arthroscopic treatment of chondromalacia patellae. J Bone Joint Surge Br 1984 Nov; 66(5):660-665.

[24] Panni AS, Tartarone M, Patricola A, Paxton EW, Fithian DC. Long-term results of lateral retinacular release. Arthroscopy 2005 May; 21(5):526-531.

[25] Alemdaroglu KB, Cimen O, Aydogan NH, Atlihan D, Iltar D. Early results of arthroscopic lateral release in patellofemoral arthritis. Knee 2008 Dec;15(6):451-455.

[26] Aglietti P, Pisaneschi A, Buzzi R, Gaudenzi A, Allegra M. Arthroscopic lateral release for patellar pain or instability. Arthroscopy 1989; 5(3): 176-183.

[27] Aderinto J, Cobb AG. Lateral release for patellofemoral arthritis. Arthroscopy 2002 April; 18(4):299-403.

[28] Dainer RD, Barrack RL, Buckley SL, Alexander AH. Arthroscopic treatment of acute patellar dislocation. Arthroscopy 1988;4(4): 267-271.

[29] Dragoo JL, Miller MD, Vaugh ZD, Schmidt JD, Handley E. Restoration of knee volume using selected arthroscopic releases. Am J Sports Med 2010 Nov;28(11):2288-2293.

[30] Kim DH, Gill TJ, Millett PJ. Arthroscopic treatment of the arthrofibrotic knee. Arthroscopy 2004 Jul; 20 Supp 2:1870194.

[31] Millett PJ, Steadman JR. The role of capsular distension in the arthroscopic management of arthrofibrosis of the knee: A technical consideration. Arthroscopy 2001;17:E31.

[32] Sprague NF 3rd, O'Connor RL, Fox JM. Arthroscopic treatment of postoperative knee fibroarthrosis. Clin Orthop Rel Res 1982 Jun;(166): 165-172.

[33] Parisien JS. The role of arthroscopy in the treatment of postoperative fibroarthrosis of the knee joint. Clin Orthop Relat Res 1988 Apr;229:1850192.

[34] Vaquero J, Vidal C, Medina E, Baena J. Arthroscopic lysis in knee arthrofibrosis. Arthroscopy 1993;9(6):691-694.

[35] Klein W, Shah N, Gassen A. Arthroscopic management of postoperative arthrofibrosis of the knee joint: indication, technique, and results. Arthroscopy 1994;10(6):591-597.

[36] Kim SJ, Choe WS. Arthroscopic findings of the synovial plicae of the knee. Arthroscopy 1997 13(1):33-41

[37] Ewing JW. Plica: pathologic or not? J Am Acad Orthop Surg 1883;1(2):117-121.

[38] Kent M, Khanduja V. Synovial plicae around the knee. Knee 2010;17(2):97-102.

[39] Weckstrom M, Niva MH, Lamminen A, Mattila VM, Pihlajamaki HK. Arthroscopic resection of medial plica of the knee in young adults. Knee 2010;17(2):103-107.

[40] Demirag B, Ozturk C, Karakayali M. Symptomatic infrapatellar plica. Knee Surg Sports Traumatol Arthrosc 2006;14(2):156-160.

[41] Boyd CR, Eakin C, Matheson GO. Infrapatellar plica as a cause of anterior knee pain. Clin J Sport Med 2005;15(2):98-103. 
[42] Bae DK, Nam GU, Sun SD, Kim YH. The clinical significance of the complete type of suprapatellar membrane. Arthroscopy 1998;14(8):830-835. 


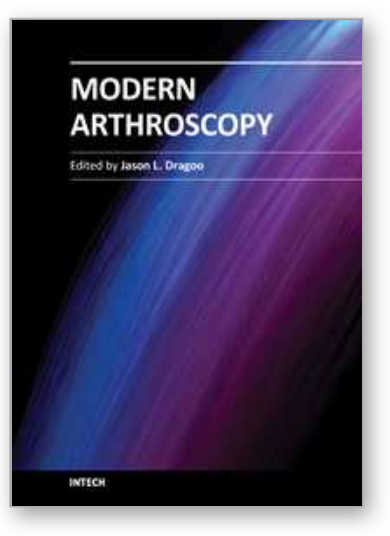

\author{
Modern Arthroscopy \\ Edited by Dr Jason L. Dragoo
}

ISBN 978-953-307-771-0

Hard cover, 302 pages

Publisher InTech

Published online 09, December, 2011

Published in print edition December, 2011

Modern Arthroscopy will assist practitioners to stay current in the rapidly changing field of arthroscopic surgery. The chapters in this book were written by a panel of international experts in the various disciplines of arthroscopy. The goals of this text are to present the classical techniques and teachings in the fields of Orthopaedics and Dentistry, but also to include new, cutting-edge applications of arthroscopy, such as temporomandibular arthroscopy and extra-articular arthroscopy of the knee, just to name a few. We hope Modern Arthroscopy becomes a core reference for your arthroscopic surgery practice.

\title{
How to reference
}

In order to correctly reference this scholarly work, feel free to copy and paste the following:

Michael R. Chen and Jason L. Dragoo (2011). Arthroscopic Soft Tissue Releases of the Knee, Modern Arthroscopy, Dr Jason L. Dragoo (Ed.), ISBN: 978-953-307-771-0, InTech, Available from: http://www.intechopen.com/books/modern-arthroscopy/arthroscopic-soft-tissue-releases-of-the-knee

\section{INTECH}

open science | open minds

\author{
InTech Europe \\ University Campus STeP Ri \\ Slavka Krautzeka 83/A \\ 51000 Rijeka, Croatia \\ Phone: +385 (51) 770447 \\ Fax: +385 (51) 686166 \\ www.intechopen.com
}

\author{
InTech China \\ Unit 405, Office Block, Hotel Equatorial Shanghai \\ No.65, Yan An Road (West), Shanghai, 200040, China \\ 中国上海市延安西路65号上海国际贵都大饭店办公楼 405 单元 \\ Phone: +86-21-62489820 \\ Fax: $+86-21-62489821$
}


(C) 2011 The Author(s). Licensee IntechOpen. This is an open access article distributed under the terms of the Creative Commons Attribution 3.0 License, which permits unrestricted use, distribution, and reproduction in any medium, provided the original work is properly cited. 